\title{
Optical Waveguiding Organic Nanorods Coated with Reversibly Switchable Fe(II) Spin Transition Nanoparticles
}

\author{
Supratim Basak, Ajay Kumar Botcha, M. Thameem Ansari, and Rajadurai Chandrasekar \\ Functional Molecular Nano/Micro Scale Laboratory, School of Chemistry, University of Hyderabad, Hyderabad 500 046, India \\ Correspondence should be addressed to Rajadurai Chandrasekar; chandrasekar100@yahoo.com
}

Received 12 July 2013; Accepted 11 September 2013

Academic Editors: K. Kalantar-Zadeh, M. Lavorgna, and M. Romero Pérez

Copyright (c) 2013 Supratim Basak et al. This is an open access article distributed under the Creative Commons Attribution License, which permits unrestricted use, distribution, and reproduction in any medium, provided the original work is properly cited.

\begin{abstract}
A dual functional nanohybrid object combining photonic and magnetic properties was successfully prepared through a "bottomup" self-assembly approach. In this method, spin transition Fe(II) coordination nanoparticles and optical wave guiding organic nanorods were generated in situ and successfully integrated together in a single pot through self-assembly. The Fe(II) nanoparticles coated on organic nanorods (nanohybrids) display temperature dependent reversible spin transition (Paramagnetic; $S=2 \leftrightarrow$ diamagnetic; $S=0$ ) behavior. The nano-hybrids show efficient optical wave guiding behavior, which demonstrates the future possibility to perform light induced excited spin state trapping (LIESST) experiments on a single spin transition nanoparticle level. These photonic and magnetic "nanohybrids" offer promising option to externally manipulate spin state of the spin transition nanoparticles using temperature as well as remote laser light.
\end{abstract}

\section{Introduction}

Bottom-up nanofabrication of self-assembled multifunctional nanoobjects and study of their uncharted physical properties at the nanoscale level are an active and sophisticated research area of nanoscience and technology [113]. Particularly programming the in situ growth of selfassembled organic and inorganic nanostructures from different molecular components and also their integration to form hybrid nanoscale objects in the same pot is a less explored area. A successful groundwork in this discipline might lead to new advanced functional hybrid nanomaterials capable of performing a sensing, electronic, photonic, and mechanical function [1-13]. For example integration of magnetically bistable inorganic nanoparticles (NPs) and optical wave guiding organic nanostructures could facilitate remote manipulation of the light sensitive spin states of individual NPs through guided laser light. Recently, we have reported for the first time on the passive wave guiding behavior of organic tubes and also demonstrated the remote electronic excitation of a mesotetratolylporphyrin nanosheet using a $20 \mu \mathrm{m}$ long optical wave guiding organic tube [14-16]. Hence we intended to study the propensity of the 1D organic solids coated with spin transition NPs [17-37] (ST-NPs) to guide source laser light under laser confocal microscope setup. It was anticipated that the dielectric difference between the 1D nano-hybrid solid and the surrounding medium would guide the optical wave along the growth axis of the waveguide.

Among NPs, Fe(II) ST-NPs are of interest due to their switchable bistable magnetic spin states between paramagnetic high-spin state $\left(S=2 ; t_{2 g}{ }^{4} e_{5}^{2}\right)$ and diamagnetic lowspin state $\left(S=0 ; t_{2 g}{ }^{6} e_{g}{ }^{0}\right)$ [17-37]. ST-NPs undergo reversible magnetic switching between low-spin (LS) and high-spin (HS) states due to the modulation of ligand field strengths depending on the temperature, light, and pressure. Till now there is no direct in situ method available on the integration of ST-NPs on other self-assembled organic nanoobjects. Recently our group reported an efficient solvent-assisted technique for the preparation of organic submicrotubes from a back-to-back coupled ditopic ligand, 4,4'-bis $(2,6-\mathrm{di}(1 \mathrm{H}$ pyrazol-1-yl)pyridin-4-yl)biphenyl, $\mathbf{L}$ in dichloromethane [12]. We found that $\mathbf{L}$ also forms crystalline organic nanorods (ONRs) in hexane. Additionally, $\mathbf{L}$ is also a good candidate for the syntheses of 1D ST metallosupramolecular coordination polymers $[38,39]$ due to its readily available two tridentate metal chelating sites on either side of the molecule. The exposure and reactivity of the chelating molecules $\mathbf{L}$ available 

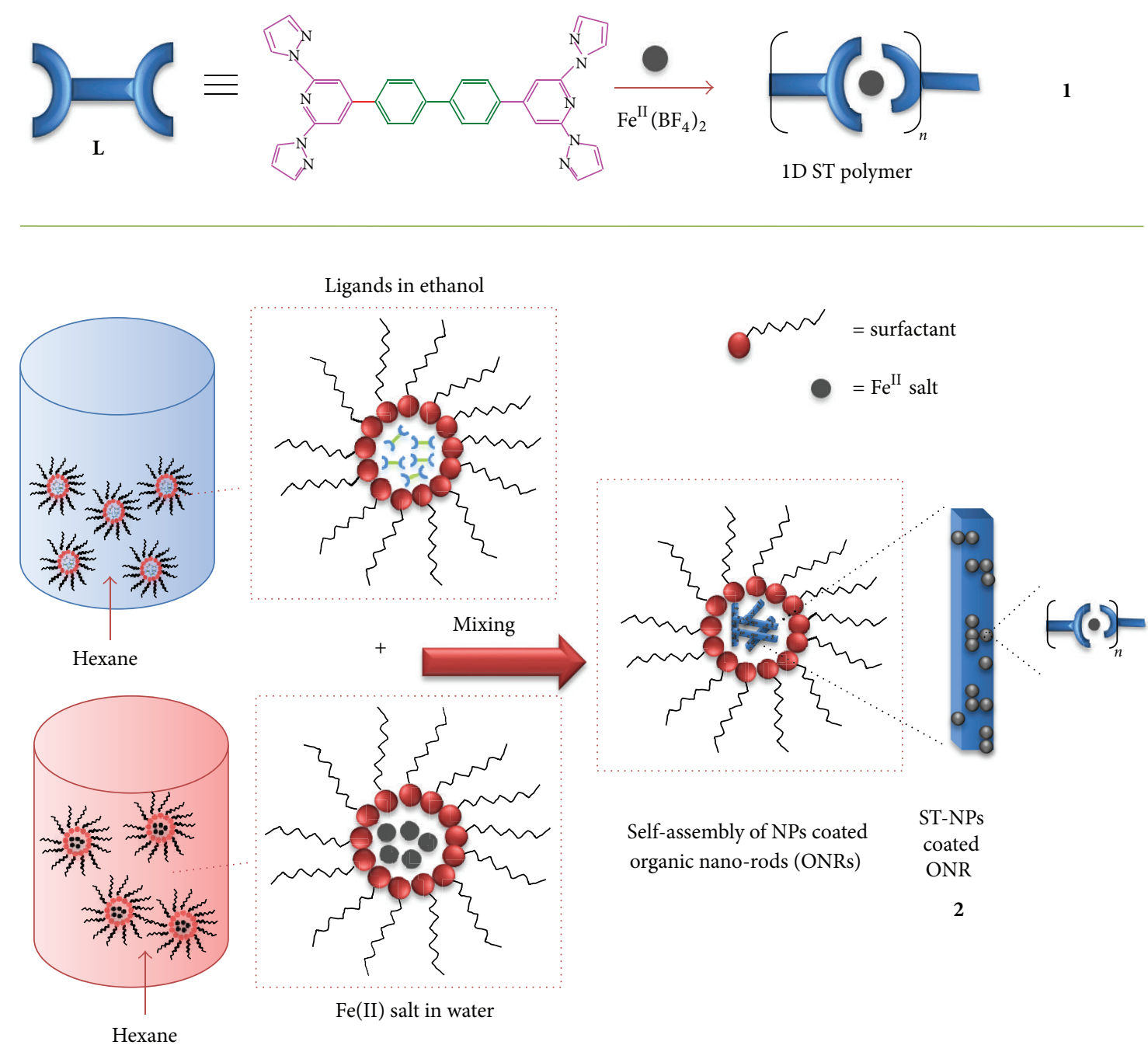

SCHEME 1: Representation of the components used in the reverse micelle technique for the fabrication of spin transition nanoparticles (STNPs) coated organic nanorods (ONRs).

on the surface of the preorganized ONRs to reactants such as $\mathrm{Fe}^{\mathrm{II}}$ ion and surfactant may also facilitate the surface selective growth of ST coordination NPs there by finally forming integrated functional ST nanohybrids.

To realize our idea, by following reverse micelle technique [40] we developed a singlestep method to fabricate ONRs from L, which are coated with ST-NPs (Scheme 1). Herein we report our nanohybrid preparation procedure: (i) selfassembly of $\mathbf{L}$ to form ONRs and (ii) self-assembly of STNPs from molecules $\mathbf{L}$ available on the surface of the ONRs in presence of $\mathrm{Fe}^{\mathrm{II}}$ salt and surfactants. We also report the electron (SEM, FESEM, and TEM), atomic force microscopy (AFM), and Raman spectroscopy images of the nanoobjects and also the variable temperature magnetic properties of bulk 1D ST coordination polymer 1 and ST-NPs coated on ONRs 2. Finally we also present our preliminary results on the optical wave guiding tendency of the nano-hybrids.

\section{Materials and Methods}

2.1. Materials. The materials citrazinicacid, $\left[\mathrm{Pd}\left(\mathrm{PPh}_{3}\right)_{4}\right]$, trifluoroacetic acid, sodium dioctyl sulphosuccinate (SDS), docosanic acid, $\mathrm{Fe}\left(\mathrm{BF}_{4}\right)_{2} \cdot 6 \mathrm{H}_{2} \mathrm{O}$, and pyrazole were purchased from Aldrich. Sodium azide, $\mathrm{KI}, \mathrm{K}_{2} \mathrm{CO}_{3}, \mathrm{NaNO}_{2}$, sodium thiosulfate, and diethyl ether were purchased from Merck chemicals. Oxalyl chloride, $\mathrm{POCl}_{3}$, iodine, and $\mathrm{NaHCO}_{3}$ were purchased from Avra Synthesis, Hyderabad. The solvents were distilled and dried before reactions and for extracting purposes. IR spectra were recorded on JASCO FT/IR-5300. Elemental analysis was recorded on a Thermo Finnigan Flash EA 1112 analyzer. Size and morphology of the nanostructures were examined by using a Philips XL30 ESEM Scanning electron microscope (SEM) using a beam voltage of $20 \mathrm{kV}$ and a Tecnai G2 FEI F12 transmission electron microscope (TEM) at an accelerating voltage of $120 \mathrm{kV}$. FESEM measurements were performed on a Hitachi 


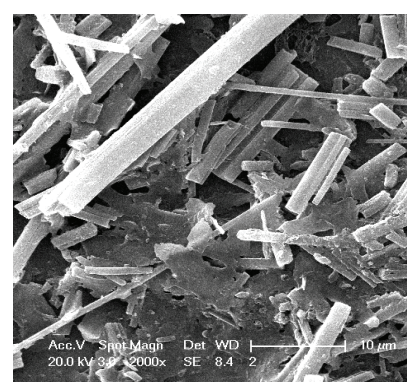

(a)

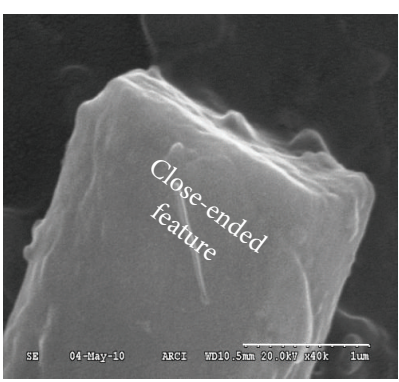

(b)

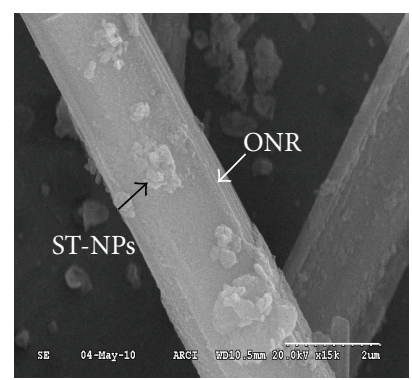

(c)

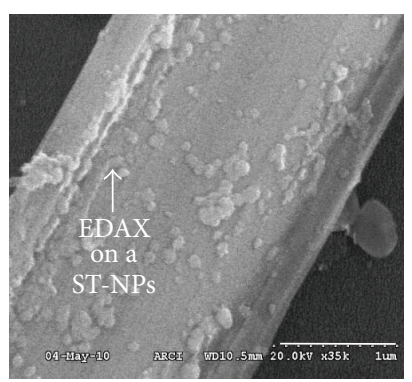

(d)

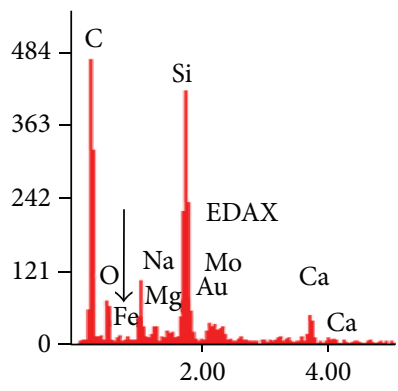

(e)

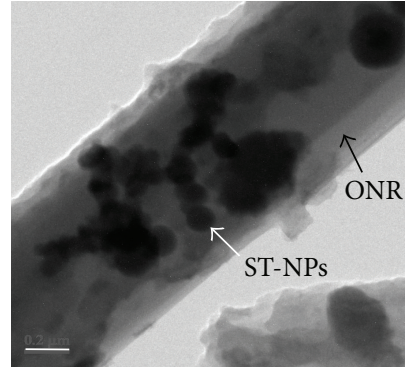

(f)

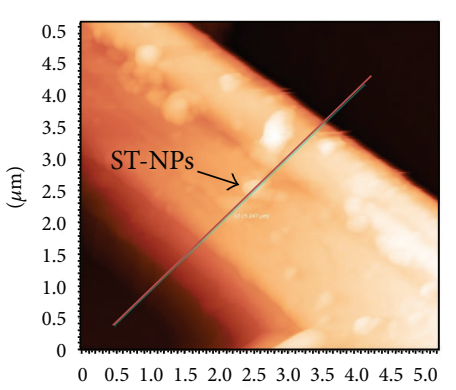

$(\mu \mathrm{m})$

(g)

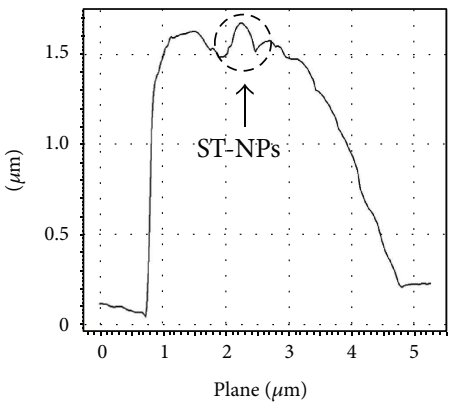

(h)

Figure 1: SEM (a), FESEM ((b)-(e)), TEM (f), and AFM ((g)-(h)) micrographs and data of ONRs coated with coordination ST-NPs 2. (a) A bunch of ONRs coated with ST-NPs; (b) close-ended feature of a ONR; ((c), (d)) a nanorod clearly shows the presence of spherical ST-NP aggregates on it; (e) energy dispersive X-ray analysis (EDAX) data collected on ST-NPs coated on ONR; (f) the dark (ST-NPs) and grey colour (ONR) contrast clearly distinguish the NPs from the ONRs; (g) AFM image of a single ONR coated with ST-NPs. (h) Cross-section profile along the red line in (g). Scale bars are: (a) $10 \mu \mathrm{m}$; (b), (c): $2 \mu \mathrm{m}$; (d) $1 \mu \mathrm{m}$; and (f) $200 \mathrm{~nm}$.

S-4500 SE/N instrument operating at $20 \mathrm{kV}$. AFM imaging was carried out on NT-MDT Model Solver Pro M microscope using semicontact mode. Variable temperature magnetic susceptibility measurements of the solid samples (1 and 2) were recorded in the temperature range of $5 \leftrightarrow 375 \mathrm{~K}$ using a Quantum Design VSM-SQUID magnetometer with an applied DC magnetic field of $1000 \mathrm{G}$. Powder sample was packed in a nonmagnetic sample holder and mounted on a nonmagnetic straw.

\subsection{Confocal Micro-Raman Spectroscopy/Waveguiding Stud-} ies. Raman spectra of the samples were recorded on a WiTec alpha 300 confocal Raman spectrometer (back scattering geometry) equipped with a Peltier-cooled CCD detector. A $400 \mathrm{~nm} \mathrm{Ar}^{+}$laser was used for the experiments. Using a 600 grooves $/ \mathrm{mm}$ grating $\mathrm{BLZ}=500 \mathrm{~nm}$, the integration time was typically $0.5000 \mathrm{~s}$. Ten accumulations were performed for acquiring a single spectrum. All measurements were collected in air and room temperature. Optical waveguiding studies were also performed on this setup.

2.3. Experimental Methods. Preparation of coordination polymer is as following (1): $40 \mathrm{mg}(0.069 \mathrm{mmol})$ of ligand 4,4'-bis(2,6-di(1H-pyrazol-1-yl)pyridin-4-yl)biphenyl $\mathbf{L}$ was dissolved in a deaerated dichloromethane $(30 \mathrm{~mL})$. To this a deaerated methanolic solution containing $35.5 \mathrm{mg}$ $(0.139 \mathrm{mmol})$ of $\mathrm{Fe}\left(\mathrm{BF}_{4}\right)_{2} \cdot 6 \mathrm{H}_{2} \mathrm{O}$ was added and stirred for 2 days under $\mathrm{N}_{2}$ atmosphere. The solvents were evaporated from the turbid solution and the obtained yellow precipitate was collected and washed with dichloromethane and methanol and finally air-dried. Yield $53 \mathrm{mg}$; FTIR $(\mathrm{KBr}$, $\left.\nu / \mathrm{cm}^{-1}\right)$ : 3524, 3435, 3128, 2924, 2854, 1631, 1572, 1523, 1460, 


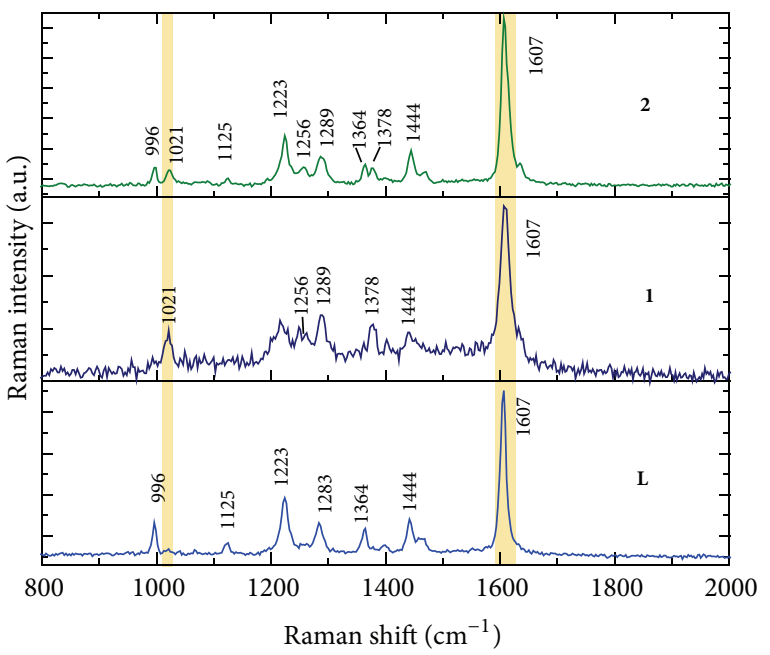

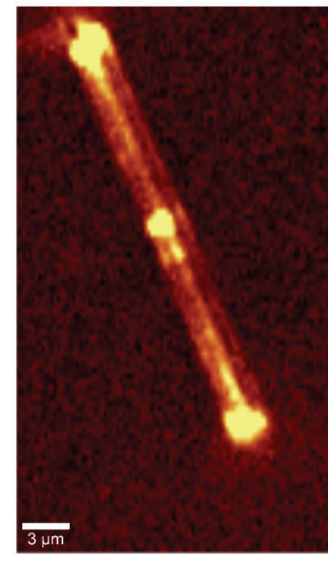

(a)

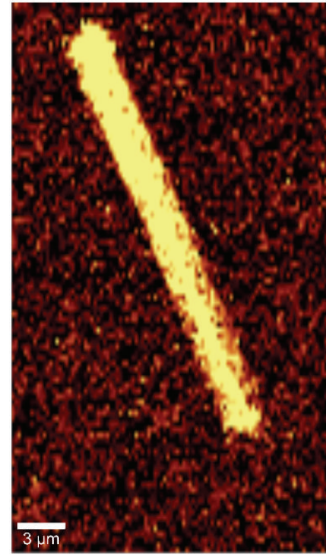

(b)

FIGURE 2: Comparative confocal Raman micro spectroscopic data collected from $\mathbf{L}, \mathbf{1}$ and the $\mathrm{Fe}^{\mathrm{II}}$ coordination nanoparticles coated organic nano-rods 2. (a) Raman image of the $1021 \mathrm{~cm}^{-1}$ region. (b) Raman image of the region between 1580 and $1631 \mathrm{~cm}^{-1}$.

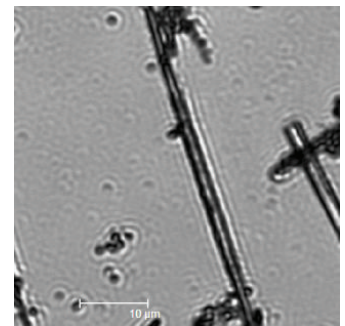

(a)

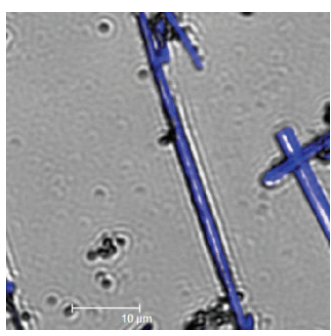

(c)

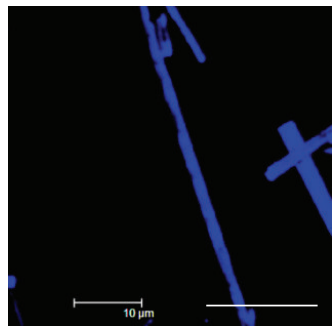

(b)

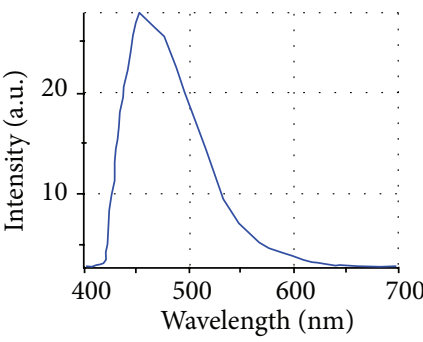

(d)
Figure 3: Confocal fluorescence microscopy data of nanorods coated with ST-NPs 2. (a) Before excitation ((b); (c)) after excitation by Ar-ion UV laser. (d) Emission spectrum of rods shown in (b) and (c).

$1406,1253,1219,1053,966,819,761$. Elemental analysis (\%) calculated for $\mathrm{C}_{34} \mathrm{H}_{24} \mathrm{~B}_{2} \mathrm{~F}_{8} \mathrm{FeN}_{10}$ : C 50.91, $\mathrm{H}$ 3.02, $\mathrm{N}$ 17.46; found C 51.41, H 2.95, N 17.25.

Preparation of ST nanoparticles coated nanorod is as follows (2): $\mathrm{Fe}\left(\mathrm{BF}_{4}\right)_{2} \cdot 6 \mathrm{H}_{2} \mathrm{O}(14.6 \mathrm{mg}, 0.664 \mathrm{mmol})$ was dissolved in water $(2 \mathrm{~mL})$, and added to a mixture of sodium dioctyl sulphosuccinate $(0.256 \mathrm{~g})$ in $10 \mathrm{~mL}$ of hexane. After a few minutes of stirring, behenic acid (39.4 mg, 1.328) was added to this suspension. 4,4'-Bis(2,6-di(1H-pyrazol1-yl)pyridin-4-yl)biphenyl (100 mg, $0.174 \mathrm{mmol}$ ) was suspended to ethanol $(10 \mathrm{~mL})$, added to the above mixture, and vigorously stirred for $36 \mathrm{~h}$. Afterwards, the mixture was evaporated to get a sticky solid, which is contaminated by surfactants, which was removed by centrifuging with ethanol for several times. The powder obtained was finally dried under vacuum to afford a yellow powder of 2 (50 mg). FTIR $\left(\mathrm{KBr}, \nu / \mathrm{cm}^{-1}\right): 3148,2918,2849,1707,1612,1572,1539,1510$, $1462,1394,1261,1207,1035,952,935,914,871,823,787,767$, $628,605,486$.

\section{Result and Discussion}

In the bulk state, treatment of $\mathbf{L}$ with $\mathrm{Fe}^{\mathrm{II}}\left(\mathrm{BF}_{4}\right)_{2}$ (1:1 stoichiometry) in acetonitrile immediately formed an insoluble yellow precipitate, which indicated the formation of a $1 \mathrm{D}$ linear coordination polymer $\left[\mathrm{Fe}^{\mathrm{II}}\left(\mathrm{L}_{2}\right)\left(\mathrm{BF}_{4}\right)_{2}\right]_{n} \mathbf{1}$. We have employed an in situ reverse micelle technique to grow selfassembled nano-hybrids, that is, ONRs coated with ST-NPs. Firstly, dispersion of an ethanol solution of $\mathbf{L}$ in hexane followed by treatment with sodium dioctyl sulfosuccinate surfactant formed micelle containing $\mathbf{L}$ stabilized by the surfactant. Secondly, dispersal of an aqueous solution of $\mathrm{Fe}^{\mathrm{II}}$ salt in hexane followed by the treatment of surfactant produced a $\mathrm{Fe}^{\mathrm{II}}$ carrying micelle stabilized by the surfactants. Mixing followed by stirring of the two solutions containing micelle carrying $\mathrm{L}$ and $\mathrm{Fe}^{\mathrm{II}}$ salt formed a yellow precipitate of ST-NPs coated ONRs 2. Filtration of the resultant precipitate followed by drying gave a light yellow powder of 2 .

The electron microscopy (SEM, FESEM, and TEM) images of 2 clearly exhibited the formation of ST-NPs on ONRs. The SEM images of $\mathbf{2}$ showed the ONRs of different lengths varying from 5-30 $\mu \mathrm{m}$ lengths and widths in the range of ca. 1-4 $\mu \mathrm{m}$ (Figure 1(a)). FESEM measurements clearly showed the growth of spherical NPs on the surface of the ONRs (Figures 1(b)-1(d)). Furthermore the energy dispersive X-ray analysis (EDAX) measurement performed on NPs coated on ONRs clearly indicated the presence of iron ions in the spherical NPs (Figure 1(e)). 


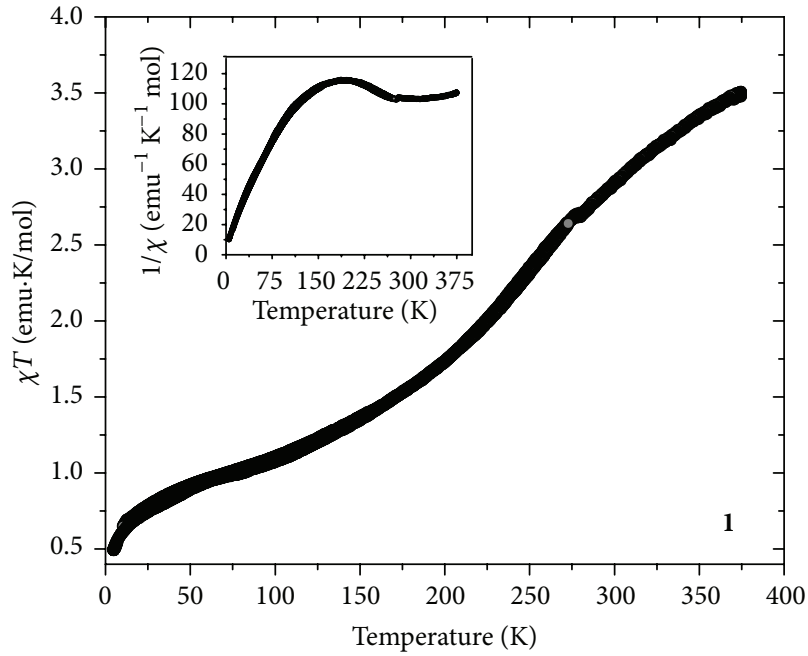

(a)

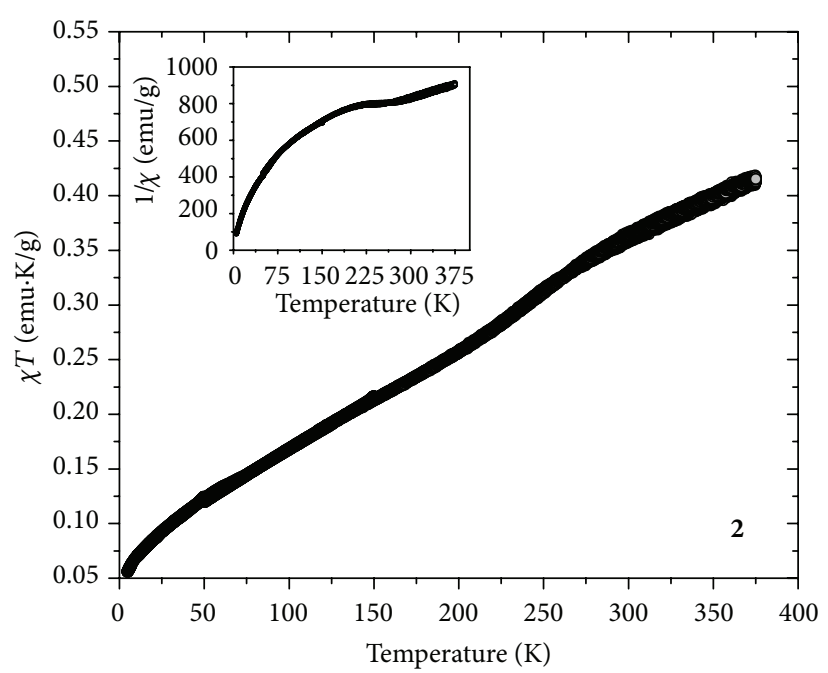

(b)

Figure 4: $\chi T=f(T)$ plots for bulk sample of 1 and the $\mathrm{Fe}^{\mathrm{II}}$ coordination nanoparticles coated organic nanorods 2 . Inset figures show the $1 / \chi$ versus $T$ plots.

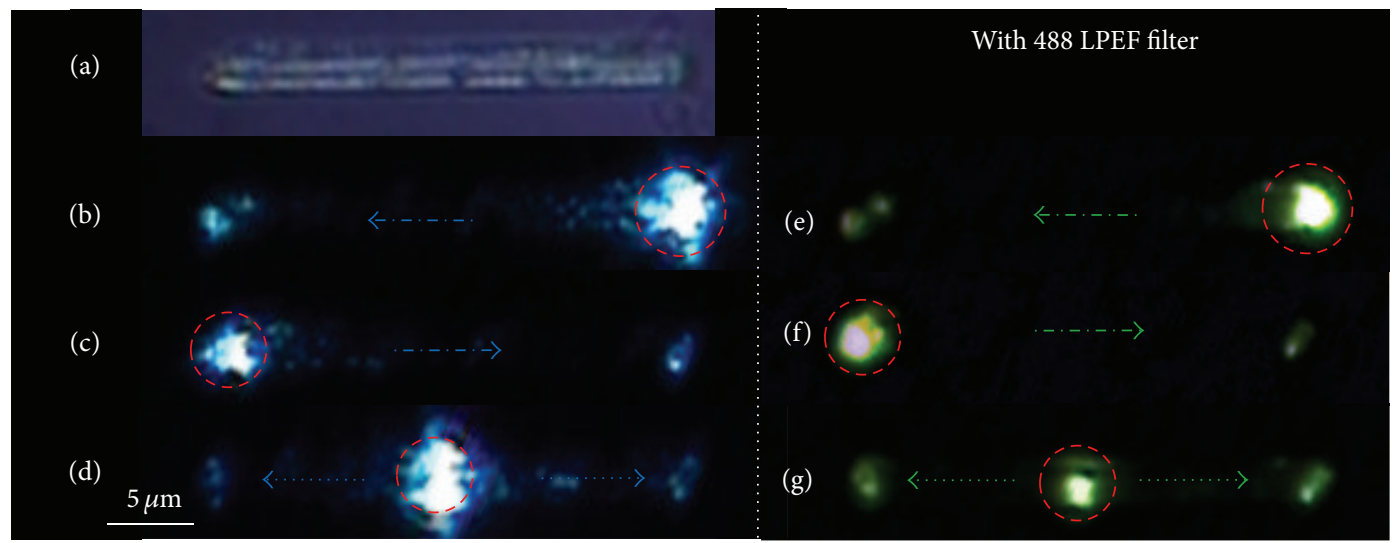

FIGURE 5: Confocal micrographs of a wave guiding ONR under $488 \mathrm{~nm} \mathrm{Ar}^{+}$laser illumination. ((a)-(d)) without $488 \mathrm{~nm}$ LPEF; ((e)-(g)) with $488 \mathrm{~nm}$ LPEF filter. Red circles show laser illumination point and blue/green arrows show the propagation direction.

Additionally AFM measurements confirmed the coassembly of ST-NPs on ONRs (Figures $1(\mathrm{~g})$ and $1(\mathrm{~h})$ ). A typical width $\times$ height range profile of selected ONRs is ca. 1-4 $\times 0.4-1.5 \mu \mathrm{m}$. Direct visualization of ST-NPs (black contrast) on the surface of the ONRs (grey contrast) was achieved by TEM measurements (Figure 1(f)). The NPs are spherical in shape and the sizes were in the range of ca. $70-200 \mathrm{~nm}$. The spherical particles were aggregated together on the surface of the ONRs. Furthermore confocal Raman

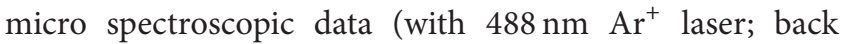
scattering mode) confirmed the presence of ST-NPs on the surface of the ONR by exhibiting characteristic Raman shifts corresponding to $\mathbf{L}$ and $\mathbf{1}$ (Figure 2). Raman imaging of a selected ONR using the NPs marker modes $992 \mathrm{~cm}^{-1}$ to $1031 \mathrm{~cm}^{-1}$ showed that the particles are mostly concentrated at the centre and at the rod ends. The confocal fluorescence microscopy studies of the nanorods coated with ST-NPs
2 displayed a blue emission at $443 \mathrm{~nm}$ demonstrating the fluorescence property of the nano-rods and also possibility to utilize these rods as active waveguides (Figure 3 ).

The temperature dependent magnetic measurements data of $\mathbf{1}$ and $\mathbf{2}$ in the range of $375 \leftrightarrow 5 \mathrm{~K}$ at 1000 Oe DC magnetic field are shown in Figure 4. For 1 the product of magnetic susceptibility and temperature $(\chi T)$ versus $T$ curve indicated the presence of a reversible ST behavior. The value of $\chi T$ at $375 \mathrm{~K}$ is ca. $3.5 \mathrm{emu} \cdot \mathrm{K} / \mathrm{mol}$, which indicated the HS state of 1 . Upon cooling down to $293 \mathrm{~K}$ the $\chi T$ value further decreased to $2.85 \mathrm{emu} \cdot \mathrm{K} / \mathrm{mol}$. This demonstrated the presence of HS and LS fractions at room temperature. Further cooling showed a steep decrease of the $\chi T$ value which reached a minimum value of ca. $0.5 \mathrm{emu} \cdot \mathrm{K} / \mathrm{mol}$ at $5 \mathrm{~K}$. This specified the completion of the ST event. The $\chi T$ curve showed a similar trend even in the heating mode performed up to $375 \mathrm{~K}$. The ST temperature of $\mathbf{1}$ is ca. $201 \mathrm{~K}$. Since 
the process of ST is very sensitive to environmental changes, to explore the ST tendency of the NPs coated on ONRs, the variable temperature magnetic properties of sample 2 were also investigated. Interestingly the reversible ST property was retained for $\mathbf{2}$ but with a change in the ST temperature (ca. $160 \mathrm{~K}$ ) and its propensity compared to 1 . The shifting of the ST to lower temperature side is perhaps due to the decrease of the long-range cooperatives in the NP state. Additionally, the origin of smooth ST compared to 1 is probably due to the presence of ST-NPs of various particle size ranges [25].

For the passive optical wave guiding studies, a visible light laser $\left(488 \mathrm{~nm} ; \mathrm{Ar}^{+}\right)$was employed to circumvent the electronic absorption of a laser light by the molecular building blocks of the rods (Figure 5(a)). The laser beam was coupled to the ONR kept on a thin glass substrate through a 50x objective $(\mathrm{NA}=0.90)$. Orthogonal illumination of a laser beam at any one of the ends of a rod (optical coupling point) showed propagation of guided laser light to the opposite end of the rod (Bright field images; Figures 5(b) and 5(c)) while irradiation of laser at the center of the rod showed guided light propagation to both ends of the rod (Figure 5(d)). The corresponding dark field images taken with a 488 long pass edge filter (LPEF) are shown in Figures 5(e)-5(g). Fe(II) STNPs are known to display light induced electron spin state trapping (LIESST) effect [41] that is, metastable high-spin state $(S=2)$ upon illumination with laser light at cryogenic temperature. In all of the reported literature works these LIESST experiments were performed on a bulk ST samples [41-44]. Our preliminary experimental results demonstrate that the LIESST studies can be done on single ST-NP level using a waveguiding organic nanorods coated with ST-NPs.

\section{Conclusions}

The presented results clearly demonstrate a successful in situ preparation of organic and inorganic nanostructures such as ST-NPs and ONRs in a single pot to create "nanohybrids." The optical wave guiding nature of the ONRs can be exploited to control the spin state of the ST-NPs using guided laser light. This is an attempt towards the "bottom-up" fabrication of externally switchable "nanohybrids."

\section{References}

[1] S. Mann, "Self-assembly and transformationof hybrid nanoobjects and nanostructures under equilibrium and nonequilibrium conditions," Nature Materials, vol. 8, pp. 781-792, 2009.

[2] C. A. Mirkin and C. M. Niemeyer, Nanobiotechnology II, WileyVCH, Weinheim, Germany, 2007.

[3] C. A. Mirkin, R. L. Letsinger, R. C. Mucic, and J. J. Storhoff, "A DNA-based method for rationally assembling nanoparticles into macroscopic materials," Nature, vol. 382, no. 6592, pp. 607609, 1996

[4] E. Katz and I. Willner, "Integrated nanoparticle-biomolecule hybrid systems: synthesis, properties, and applications," Angewandte Chemie International Edition, vol. 43, pp. 6042-6108, 2004.
[5] T. Nakanishi, "Supramolecular soft and hard materials based on self-assembly algorithms of alkyl-conjugated fullerenes," Chemical Communications, vol. 46, no. 20, pp. 3425-3436, 2010.

[6] K. J. C. van Bommel, A. Friggeri, and S. Shinkai, "Organic templates for the generation of inorganic materials," Angewandte Chemie International Edition, vol. 42, no. 9, pp. 980-999, 2003.

[7] M. Li, C. Viravaidya, and S. Mann, "Polymer-mediated synthesis of ferritin-encapsulated inorganic nanoparticles," Small, vol. 3, no. 9, pp. 1477-1481, 2007.

[8] M. Okuda, Y. Kobayashi, K. Suzuki et al., "Self-organized inorganic nanoparticle arrays on protein lattices," Nano Letters, vol. 5, no. 5, pp. 991-993, 2005.

[9] P. J. Meadows, E. Dujardin, S. R. Hall, and S. Mann, "Templatedirected synthesis of silica-coated j-aggregate nanotapes," Chemical Communications, no. 29, pp. 3688-3690, 2005.

[10] A. J. Patil, M. Li, E. Dujardin, and S. Mann, "Novel bioinorganic nanostructures based on mesolamellar intercalation or singlemolecule wrapping of DNA using organoclay building blocks," Nano Letters, vol. 7, no. 9, pp. 2660-2665, 2007.

[11] M. Sofos, J. Goldberger, D. A. Stone et al., "A synergistic assembly of nanoscale lamellar photoconductor hybrids," Nature Materials, vol. 8, no. 1, pp. 68-75, 2009.

[12] N. Chandrasekhar and R. Chandrasekar, "Engineering selfassembled fluorescent organic nanotapes and submicrotubes from n-conjugated molecules," Chemical Communications, vol. 46, no. 17, pp. 2915-2917, 2010.

[13] S. Basak and R. Chandrasekar, "Multiluminescent hybrid organic/inorganic nanotubular structures: one-pot fabrication of tricolor (Blue Red-Purple) luminescent parallepipedic organic superstructure grafted with europium complexes," Advanced Functional Materials, vol. 21, no. 4, pp. 667-673, 2011.

[14] N. Chandrasekhar, M. A. Mohiddon, and R. Chandrasekar, "Organic submicro tubular optical waveguides: self-assembly, diverse geometries, efficiency, and remote sensing properties," Advanced Optical Materials, vol. 1, no. 4, pp. 305-311, 2013.

[15] P. Hui and R. Chandrasekar, "Light propagation in highspin organic microtubes self-assembled from shape persistent macrocycles carrying oxo-verdazyl biradicals," Advanced Materials, vol. 25, no. 21, pp. 2963-2967, 2013.

[16] N. Chandrasekhar and R. Chandrasekar, "Reversibly shapeshifting organic optical waveguides: formation of organic nanorings, nanotubes, and nanosheets," Angewandte Chemie International Editio, vol. 51, no. 15, pp. 3556-3561, 2012.

[17] P. Gütlich and H. A. Goodwin, Eds., Spin Crossover in Transition Metal Compounds I, vol. 233 of Topics in Current Chemistry, Springer, Berlin, Germany, 2004.

[18] H. Spiering, Spin Crossover in Transition Metal Compounds III, vol. 235 of Topics in Current Chemistry, Springer, Berlin, Germany, 2004.

[19] E. König, "Structural changes accompanying continuous and discontinuous spin-state transitions," in Progress in Inorganic Chemistry, vol. 35, p. 527, 1987.

[20] A. B. Gaspar, V. Ksenofontov, M. Seredyuk, and P. Gütlich, "Multifunctionality in spin crossover materials," Coordination Chemistry Reviews, vol. 249, no. 23, pp. 2661-2676, 2005.

[21] P. Gütlich, Y. Garcia, and T. Woike, "Photoswitchable coordination compounds," Coordination Chemistry Reviews, vol. 219221, pp. 839-879, 2001.

[22] M. A. Halcrow, "Iron(II) complexes of 2,6-di(pyrazol-1yl)pyridines-a versatile system for spin-crossover research," Coordination Chemistry Reviews, vol. 253, no. 21-22, pp. 24932514, 2009. 
[23] T. Forestier, S. Mornet, N. Daro et al., "Nanoparticles of iron(II) spin-crossover," Chemical Communications, no. 36, pp. 43274329, 2008.

[24] E. Coronado, J. R. Galán-Mascarós, M. Monrabal-Capilla, J. Garca-Martnez, and P. Pardo-Ibáñez, "Bistable spin-crossover nanoparticles showing magnetic thermal hysteresis near room temperature," Advanced Materials, vol. 19, pp. 1359-1361, 2007.

[25] F. Volatron, L. Catala, E. Rivière, A. Gloter, O. Stéphan, and T. Mallah, "Spin-crossover coordination nanoparticles," Inorganic Chemistry, vol. 47, no. 15, pp. 6584-6586, 2008.

[26] I. Boldog, A. B. Gaspar, V. Martinez et al., "Spin-crossover nanocrystals with magnetic, optical, and structural bistability near room temperature," Angewandte Chemie International Edition, vol. 47, no. 34, pp. 6433-6437, 2008.

[27] J. Larionova, L. Salmon, Y. Guari et al., "Towards the ultimate size limit of the memory effect in spin-crossover solids," Angewandte Chemie International Edition, vol. 47, no. 43, pp. 8236-8240, 2008.

[28] J.-F. Létard, "Photomagnetism of iron(II) spin crossover complexes-the T(LIESST) approach," Journal of Materials Chemistry, vol. 16, pp. 2550-2559, 2006.

[29] R. Chandrasekar, F. Schramm, O. Fuhr, and M. Ruben, "An iron(II) spin-transition compound with thiol anchoring groups," European Journal of Inorganic Chemistry, no. 17, pp. 2649-2653, 2008.

[30] M. Cavallini, I. Bergenti, S. Milita et al., "Micro and nanopatterning of spin-transition compounds into logical structures," Angewandte Chemie International Edition, vol. 47, no. 45, pp. 8596-8600, 2008.

[31] C. Rajadurai, F. Schramm, S. Brink et al., "Spin transition in a chainlike supramolecular iron(II) complex," Inorganic Chemistry, vol. 45, no. 25, pp. 10019-10021, 2006.

[32] C. Rajadurai, O. Fuhr, R. Kruk, M. Ghafari, H. Hahn, and M. Ruben, "Above room temperature spin transition in a metallo-supramolecular coordination oligomer/polymer," Chemical Communications, no. 25, pp. 2636-2638, 2007.

[33] C. Rajadurai, Z. Qu, O. Fuhr et al., "Lattice-solvent controlled spin transitions in iron(ii) complexes," Dalton Transactions, no. 32, pp. 3531-3537, 2007.

[34] N. Chandrasekhar and R. Chandrasekar, "Super hybrid tridentate ligands': 4-substituted-2-(1-butyl-1H-1,2,3-triazol-4-yl)-6(1H-pyrazol-1-yl)pyridine ligands coordinated to $\mathrm{Fe}(\mathrm{ii})$ ions display above room temperature spin transitions," Dalton Transactions, vol. 39, no. 41, pp. 9872-9878, 2010.

[35] S. Basak, P. Hui, and R. Chandrasekar, "Flexible and optically transparent polymer embedded nano/micro scale spin crossover Fe(II) complex patterns/arrays," Chemistry of Materials, vol. 25, no. 17, pp. 3408-3413, 2013.

[36] I. Salitros, J. Pavlik, R. Boca, O. Fuhr, C. Rajadurai, and M. Ruben, "Supramolecular lattice-solvent control of iron(ii) spin transition parameters," Crystal Engineering Communications, vol. 12, no. 8, pp. 2361-2368, 2010.

[37] R. Gonzalez-Prieto, B. Fleury, F. Schramm et al., "Tuning the spin-transition properties of pyrene-decorated 2,6bispyrazolylpyridine based Fe(ii) complexes," Dalton Transactions, vol. 40, no. 29, pp. 7564-7570, 2011.

[38] N. Chandrasekhar and R. Chandrasekar, “Click-fluors': synthesis of a family of $\pi$-conjugated fluorescent back-to-back coupled 2,6-bis(triazol-1-yl)pyridines and their self-assembly studies," Journal of Organic Chemistry, vol. 75, pp. 4852-4855, 2010.
[39] S. Basak, P. Hui, S. Boodida, and R. Chandrasekar, "Micropatterning of metallopolymers: syntheses of back-to-back coupled octylated 2,6-Bis(pyrazolyl)pyridine ligands and their solutionprocessable coordination polymers," Journal of Organic Chemistry, vol. 77, no. 7, pp. 3620-3626, 2012.

[40] S. Vaucher, M. Li, and S. Mann, "Synthesis of prussian blue nanoparticles and nanocrystal superlattices in reverse microemulsion," Angewandte Chemie International Edition, vol. 39, no. 10, pp. 1793-1796, 2000.

[41] P. Chakraborty, M. L. Boillot, A. Tissot, and A. Hauser, "Photoinduced relaxation dynamics in iron(ii) spin-crossover nanoparticles: the significance of crystallinity," Angewandte Chemie International Edition, vol. 52, no. 1, p. 1, 2013.

[42] S. Decurtins, P. Gütlich, C. P. Köhler, and H. Spiering, "New examples of light-induced excited spin state trapping (liesst) in iron(ii) spin-crossover systems," Journal of the Chemical Society, Chemical Communications, no. 7, pp. 430-432, 1985.

[43] J.-F. Létard, P. Guionneau, L. Rabardel et al., "Structural, magnetic, and photomagnetic studies of a mononuclear iron(II) derivative exhibiting an exceptionally abrupt spin transition. light-induced thermal hysteresis phenomenon," Inorganic Chemistry, vol. 37, no. 17, pp. 4432-4441, 1998.

[44] N. O. Moussa, E. Trzop, S. Mouri et al., "Wavelength selective light-induced magnetic effects in the binuclear spin crossover compound $\left[\mathrm{Fe}(\mathrm{bt})(\mathrm{NCS})_{2}\right]_{2}$ (bpym)," Physical Review B, vol. 75, no. 5, Article ID 054101, 2007. 

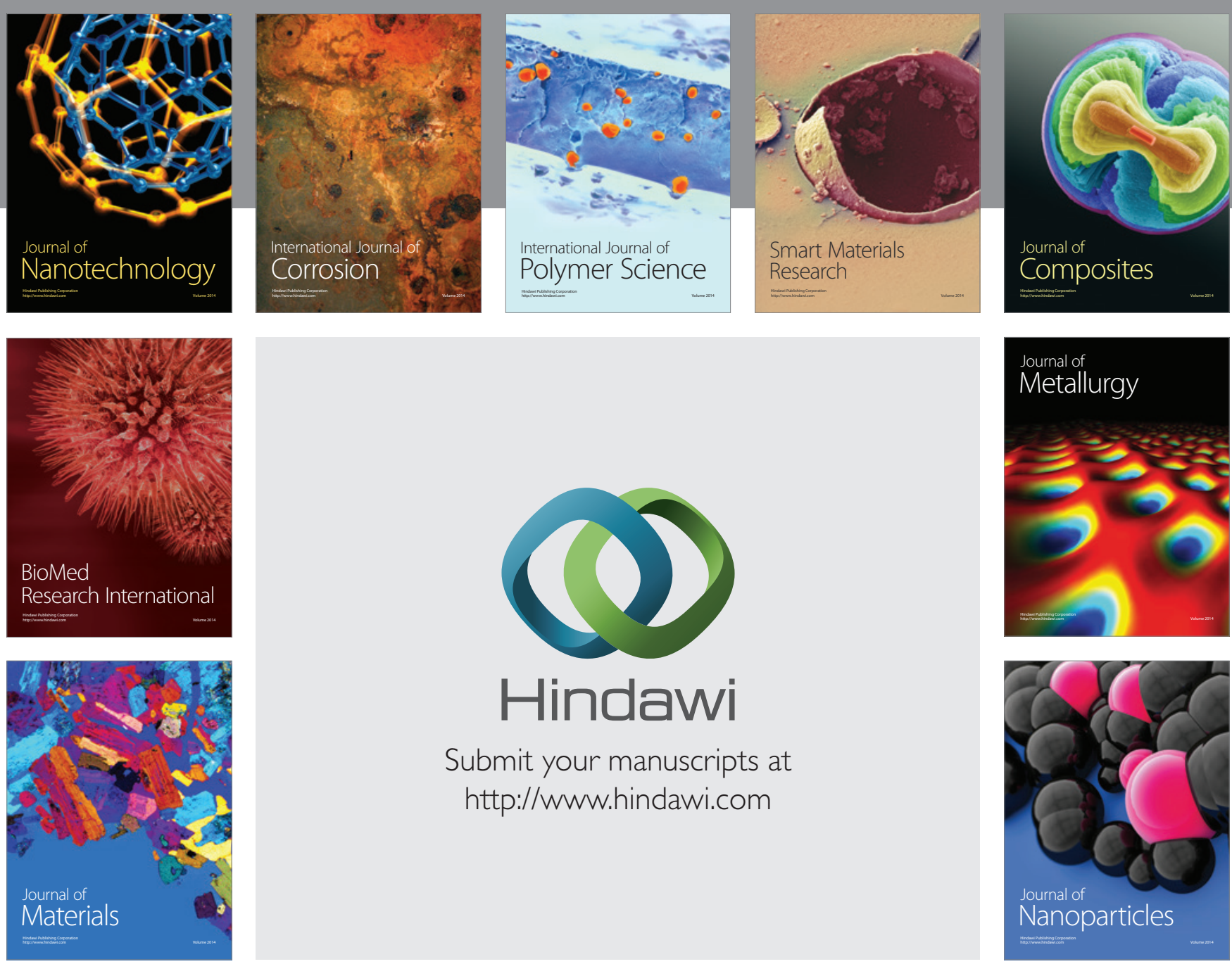

Submit your manuscripts at http://www.hindawi.com
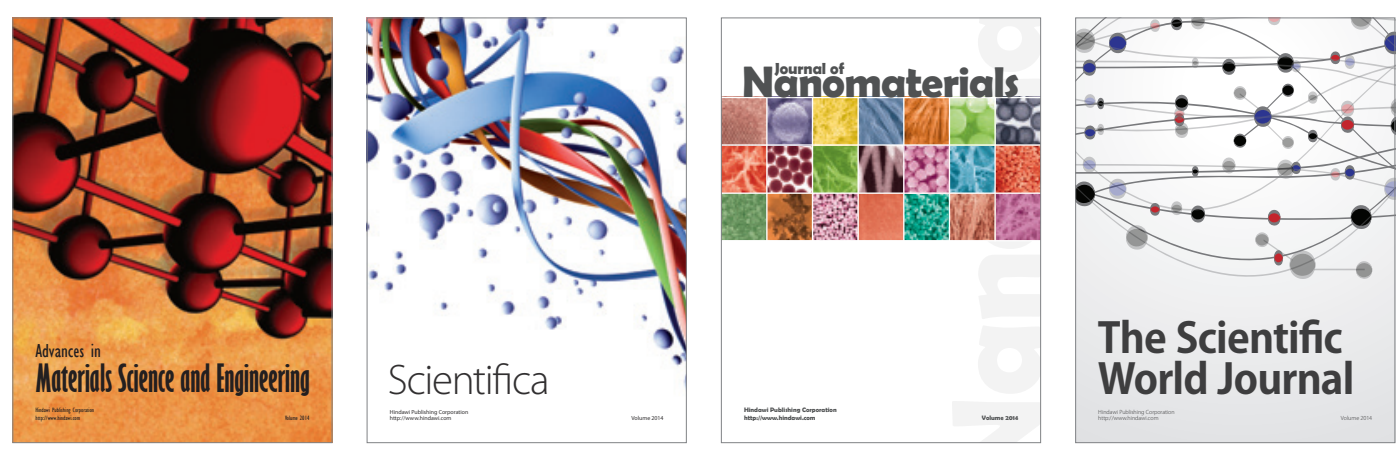

\section{The Scientific World Journal}
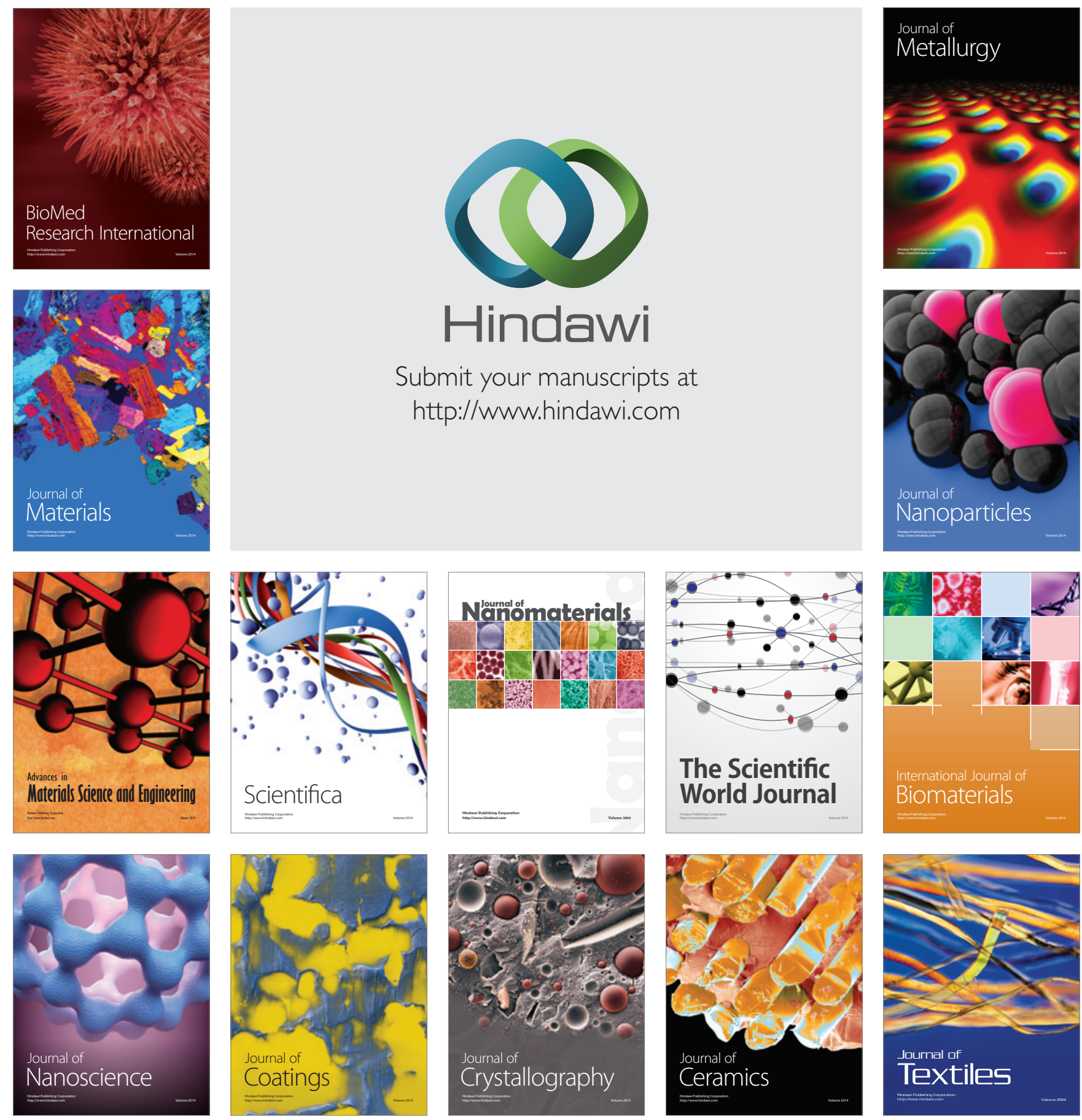